\title{
Chiral Asymmetry from a 5D Higgs Mechanism
}

\author{
Alberto Salvid] and Mikhail Shaposhnikov2 \\ Institut de Théorie des Phénomènes Physiques, \\ École Polytechnique Fédérale de Lausanne, \\ CH-1015 Lausanne, Switzerland
}

\begin{abstract}
An intriguing feature of the Standard Model is that the representations of the unbroken gauge symmetries are vector-like whereas those of the spontaneously broken gauge symmetries are chiral. Here we provide a toy model which shows that a natural explanation of this property could emerge in higher dimensional field theories and discuss the difficulties that arise in the attempt to construct a realistic theory. An interesting aspect of this type of models is that the $4 \mathrm{D}$ low energy effective theory is not generically gauge invariant. However, the non-invariant contributions to the observable quantities are very small, of the order of the square of the ratio between the light particle mass scale and the Kaluza-Klein mass scale. Remarkably, when we take the unbroken limit both the chiral asymmetry and the non-invariant terms disappear.
\end{abstract}

\footnotetext{
${ }^{1}$ Email: alberto.salvio@epfl.ch

${ }^{2}$ Email: Mikhail.Shaposhnikov@epfl.ch
} 


\section{Contents}

1 Introduction $\quad 2$

2 Basic Idea 4

2.1 Review of $5 \mathrm{D}$ fermions on domain walls . . . . . . . . . . 5

2.2 Simple example: $5 \mathrm{D}$ fermion on $S^{1} \ldots \ldots \ldots \ldots \ldots$

2.3 Infinite fifth dimension . . . . . . . . . . . . . . . . . . . . . . 7

3 A Bosonic Completion: 5D warped Higgs Model 10

3.1 Gauge fixing and perturbations . . . . . . . . . . . . . 11

3.2 Spin-1 sector and coupling with fermions . . . . . . . . . . . 12

3.3 Spin-0 sector . . . . . . . . . . . . . . . . . 14

3.4 Counting the degrees of freedom . . . . . . . . . . . . . . 16

4 4D Effective Theory and Gauge Invariance 17

4.1 Small explicit breaking of gauge invariance . . . . . . . . . . . . . . . 18

$4.25 \mathrm{D}$ versus $4 \mathrm{D}$ Higgs mechanism . . . . . . . . . . . . . . . . . 20

5 Non-Abelian Extensions

5.1 General non-Abelian gauge groups . . . . . . . . . . . . . . . . . . . . . . . . .

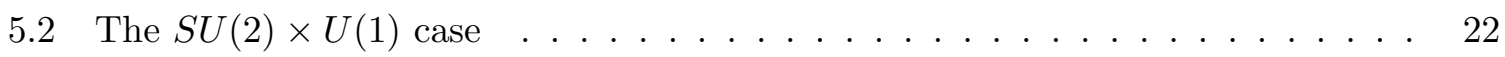

6 Conclusions and Outlook $\quad \lcm{24}$

A $R_{\xi}$ Gauges in 5D Warped Models $\quad 26$

B Higher Dimensional Operators $\quad 27$

\section{Introduction}

One of the main topics in the Physics-Beyond-the-Standard-Model is the attempt to understand fermion representations. From this point of view, models with extra dimensions are useful as they can lead to chiral 4D effective theories, both in standard Kaluza-Klein (KK) scenarios [1] and in brane worlds [2]. In some cases, in addition to a chiral spectrum, one can also obtain tachyonic scalar fields [3] that can be candidates for the Higgs field in 4 dimensions. These properties are intriguing as a natural question is whether or not there exists a more fundamental relation between the chiral asymmetry, which we observe in our world, and the Higgs mechanism.

The answer to such a question would also help us to understand the structure of the Standard Model (SM), which actually possesses the following very special feature: vector-like representations always correspond to unbroken gauge symmetries, that is the electromagnetic and the color gauge symmetries, whereas chiral representations are associated to the broken part of the SM gauge group. Is this just a coincidence or is there a deep reason? 
In the present paper we argue that there could be an explanation for that in the framework of higher dimensional models. More precisely we construct a simple 5D model in which a 4D chiral asymmetry and the Higgs mechanism are related in a way that, when we continuously turn on the Higgs order parameter, a vector-like fermion representation becomes chiral.

This property emerges quite naturally in theories where left-handed and right-handed fermions are (dynamically) localized on two different branes. In this type of constructions, one important theoretical issue is the gauge invariance of the $4 \mathrm{D}$ effective theory. Indeed, if a chiral asymmetry appears in the 4D effective theory, it may happen to have a gauge anomaly. However, we leave the study of anomalies for a future work and concentrate here on a semiclassical approximation 3 .

In fact, even the purely bosonic sector of the low energy theory can appear to be not gauge invariant. We address this problem by considering a simple bosonic completion, which includes an Abelian gauge field and a charged scalar. Our 5D bosonic action also includes some weight functions that may have their origin in warped compactifications of more fundamental theories [4, 5, 6. By considering a simple set up for the weight functions, we prove that, in the case in which the $5 \mathrm{D}$ gauge symmetry is broken, the action for the light modes generally cannot be written as a gauge invariant action (with at most a spontaneous breaking of the gauge invariance). However, we also show that the values of the observable quantities are extremely close to the predictions of a gauge invariant theory. When the 5D gauge symmetry is restored, the $4 \mathrm{D}$ effective theory acquires an exact gauge symmetry and, remarkably, this happens when the fermion representation becomes vector-like and a gauge anomaly cannot appear.

Here, we also comment on the nature of the Higgs mechanism that is used to achieve the main purpose of the paper: we clarify that, in our model, it is not possible to study the Higgs mechanism directly in the $4 \mathrm{D}$ effective theory and we provide some link to a previous work on this topic [7]. This seems a very important point that one should keep in mind in performing dimensional reductions, where it is usually assumed that tachyons in the $4 \mathrm{D}$ effective theory can consistently trigger the spontaneous symmetry breaking. Indeed, our 5D model represents a counter example for such a procedure.

Finally, we discuss non-Abelian generalizations of the simple aforementioned $U(1)$ model. In particular we discuss the $S U(2) \times U(1)$ case and describe our mechanism in a model that resembles the electroweak theory in the low energy limit. However, it is unclear if a complete phenomenological description of the masses and the interactions of the observed particles can be achieved in this type of models. Indeed, in the minimal set up that we consider in the paper the correct interactions of the $\mathrm{Z}$ vector boson cannot be reproduced and it seems quite difficult to achieve realistic fermion and vector boson masses.

The paper is organized as follows. In Section 2 we explain in more detail our basic idea and we study a 5D spinor field coupled to a background gauge field and a domain wall configuration. Then, in Section 3 we provide a simple bosonic completion in which the gauge field is promoted to a dynamical field associated to a (spontaneously broken) gauge symmetry. Therefore, we are ready to turn in Section 4 to the study of the gauge invariance of the $4 \mathrm{D}$ effective theory. In Section 5 we study the non-Abelian extensions. Finally, in Section 6 we provide the conclusions

\footnotetext{
${ }^{3}$ Here semiclassical approximation means that loop contributions have not been included in deriving the 4D effective theory.
} 
and some outlook. We leave to the appendix a technical discussion on the gauge fixing.

\section{Basic Idea}

In this section we explain how a relation between the Higgs mechanism and the low energy chiral asymmetry can occur. We would like to construct a model in which exact low energy gauge symmetry corresponds to a vector-like spectrum whereas the broken phase is associated to a chiral one. Here we show that this idea can be implemented by starting with a higher dimensional model.

As a matter of fact the $4 \mathrm{D}$ gauge fields coming from dimensional reductions have generally a constant wave function along the internal dimensions. In the presence of a higher dimensional Higgs mechanism, these gauge fields become massive and in general acquire a non-trivial profile

along the extra dimensions, which can be peaked

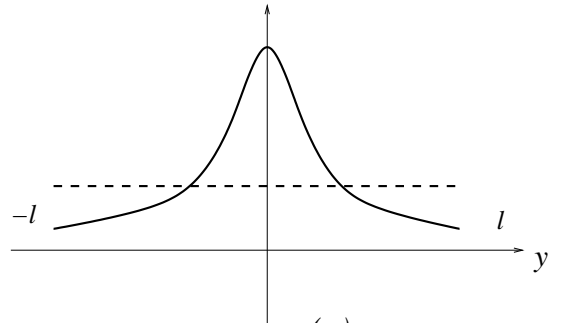

(a)

around some particular points. Now if fermions with a given chirality, say left-handed fermions, are localized on these points, whereas the righthanded modes are suppressed there, the low energy theory will certainly present a chiral asymmetry.

For the sake of definiteness, let us discuss now a simple $5 \mathrm{D}$ case with an $S^{1}$ internal space. Therefore, the extra dimension $y$ is subjected to the equivalence condition $y \sim y+2 l$, where $l / \pi$ is the radius of $S^{1}$. Without loss of generality we focus on the region $-l \leq y \leq l$. In Plot $(a)$ we give the $4 \mathrm{D}$ vector field profile along the fifth dimension in two cases: in the broken phase (continuous line) and in the unbroken phase (dashed line). As we have pointed out, this function is constant in the latter case, but is assumed to be peaked on some point - here $y=0$ - in the former case. Then, in Plot (b) we present a simple step function domain wall configuration $\varphi$, which can localize fermions with different chiralities on different points of the fifth dimension [10, 11]; this is actually a two domain wall configuration as the extra dimension has a period $2 l$. Finally, in Plot $(c)$, we show the left-handed zero mode profile (continuous line) and the right-handed one

\footnotetext{
${ }^{4} \mathrm{An}$ exception is given by $4 \mathrm{D}$ gauge fields corresponding to the isometry group of the internal space [8], but here we only consider $4 \mathrm{D}$ vectors descending from higher dimensional gauge fields and orthogonal to the gauge field background. In this case it can be proved, by using the formalism of 9 , that they do not mix with the spin-1 fields coming from gravity and that they have constant profiles if the gauge symmetry is unbroken.
} 
(dashed line), which Configuration (b) leads to. As we can see, the left-handed mode is peaked on $y=0$, whereas the right-handed mode is peaked on $y= \pm l$, which actually represents a single point because the extra dimension is periodic. Since the effective coupling constants between the fermions and the vector field are given by the overlap integral between the function in Plot $(a)$ and the absolute value squared of the fermion profiles in Plot $(c)$, we expect a $4 \mathrm{D}$ chiral asymmetry in the broken case, which should disappear when we take the unbroken limit.

We will return to this simple example in Subsection 2.2, where we will render the discussion more quantitative. Moreover, in Subsection 2.3 we shall prove that our basic idea can be also implemented in the presence of an infinite extra dimension and, therefore, is not based on the fact that the internal space is compact.

Here we also stress that some ingredients that we used in this section are well-known in the literature of higher dimensional model building: the fact that a non-trivial profile of the light gauge fields can emerge from a Higgs mechanism and modify the $4 \mathrm{D}$ effective coupling constants have already been found [12. As an original part here we establish that this effect can actually be applied to find a relation between the Higgs mechanism and the low energy chiral asymmetry.

\subsection{Review of 5D fermions on domain walls}

In this subsection we review the basics of $5 \mathrm{D}$ fermions on domain walls in order to fix our conventions and to quantitatively implement our idea.

The simplest set up for the fermion action $S_{F}$ to realize our mechanism consists of a $5 \mathrm{D}$ spinor $\psi$ and a domain wall configuration $\varphi$, which traps $4 \mathrm{D}$ fermions with different chiralities in different points of the fifth dimension. Therefore, we write [2]

$$
S_{F}=\int d^{5} X\left(\bar{\psi} \Gamma^{M} D_{M} \psi+\varphi \bar{\psi} \psi\right)
$$

where $\varphi$ is assumed to be a non dynamical field, which depends on the fifth dimension $y$, but is independent of the $4 \mathrm{D}$ coordinate $x$, and $\Gamma^{M}$ are the $5 \mathrm{D}$ gamma matrices $(M=0,1,2,3, y)$. Moreover, the covariant derivative $D_{M} \psi$ is defined by $D_{M} \psi=\left(\partial_{M}+i e_{f} A_{M}\right) \psi$, where $A_{M}$ is a dynamical 5D gauge field and $e_{f}$ the corresponding fermion charge. Action (2.1) corresponds to the following equation of motion (EOM):

$$
\Gamma^{M} D_{M} \psi+\varphi \psi=0 .
$$

We are now interested in the linear version of (2.2) with respect to the dynamical fields, in order to extract information on the fermion spectrum. By assuming the vacuum expectation value (VEV) of $A_{M}$ equal to zero, this linearization is

$$
\left(\not \partial+\gamma^{5} \partial_{y}+\varphi\right) \psi=0,
$$

where $\not \partial \equiv \gamma^{\mu} \partial_{\mu}$ and we have introduced the $4 \mathrm{D}$ gamma matrices $\gamma^{\mu} \equiv \Gamma^{\mu}$ and the $4 \mathrm{D}$ chirality matrix $\gamma^{5} \equiv \Gamma^{y}$. We can now proceed in a standard way and project (2.3) onto the left-handed and right-handed subspaces:

$$
\not \partial \psi_{R}+\left(\partial_{y}+\varphi\right) \psi_{L}=0, \quad \not \partial \psi_{L}+\left(-\partial_{y}+\varphi\right) \psi_{R}=0,
$$


where we used $\gamma^{5} \psi_{L, R}= \pm \psi_{L, R}$. We want now to study the $4 \mathrm{D}$ spectrum and, therefore, we perform a KK decomposition as follow: 5 :

$$
\psi_{L, R}(x, y)=\sum_{n} \psi_{L, R}^{(n)}(x) f_{L, R}^{(n)}(y)
$$

From (2.4) it is trivial to obtain and solve the equations for the zero modes, which are defined by $\not \partial \psi_{L, R}^{(0)}=0$. We have

$$
f_{L}^{(0)}(y) \propto \exp \left[-\int^{y} d y^{\prime} \varphi\left(y^{\prime}\right)\right], \quad f_{R}^{(0)}(y) \propto \exp \left[+\int^{y} d y^{\prime} \varphi\left(y^{\prime}\right)\right] .
$$

On the other hand, the equations for the massive modes, which satisfy $\not \partial \psi_{L, R}^{(n)}=M_{n} \psi_{R, L}^{(n)}$, are

$$
\left(-\partial_{y}^{2}+V_{L, R}\right) f_{L, R}^{(n)}=M_{n}^{2} f_{L, R}^{(n)}
$$

which are Schroedinger equations with potentials $V_{L, R}=\mp \partial_{y} \varphi+\varphi^{2}$. We can focus only on the Schroedinger equation for one chirality, say the left-handed wave functions $f_{L}^{(n)}$, because the right-handed counterpart can be obtained by using the relation $M_{n} f_{R}^{(n)}=-\left(\partial_{y}+\varphi\right) f_{L}^{(n)}$, which also follows from (2.4). The hamiltonians that appear on the left hand side of (2.7) are hermitian. This property can be proved by deriving the EOM from the action and by requiring that the boundary terms, which come from the integration by parts, vanish.

\subsection{Simple example: 5D fermion on $S^{1}$}

We now return to the simple set up that we have qualitatively discussed at the beginning of this section with the help of Plots $(a),(b)$ and $(c)$. As an original discussion, in this subsection we quantitatively study how a low energy chiral asymmetry and the spontaneous symmetry breaking (SSB) of the gauge symmetry can emerge from a single mechanism in this simple case.

We observe that Plot $(b)$ corresponds to the configuration $\varphi(y)=h(2 \theta(y)-1)$, where $h$ is a positive constant and $\theta(y)=1$ for $y>0$ and $\theta(y)=0$ for $y<0$. This domain wall background leads to the following left-handed and right-handed zero modes wave functions:

$$
\begin{aligned}
f_{L}^{(0)}(y) & =\sqrt{\frac{h}{1-e^{-2 h l}}} \exp \{h[\theta(-y)-\theta(y)] y\}, \\
f_{R}^{(0)}(y) & =\sqrt{\frac{h}{1-e^{-2 h l}}} \exp \{-h l+h[\theta(y)-\theta(-y)] y\},
\end{aligned}
$$

where we have used Eqs. (2.6) and the normalization constants have been computed in a way that the kinetic terms for $\psi_{L, R}^{(0)}$ are canonically normalized. We notice that both the zero modes satisfy the $S^{1}$ boundary conditions, and that the discontinuity of $\varphi$ on $y=0$ and $y=l$ induces a discontinuity of the derivatives of both the zero modes there.

\footnotetext{
${ }^{5}$ In principle $n$ can be a discrete or a continuous variable.

${ }^{6}$ We remind that we have focused on the region $-l \leq y \leq l$ without loss of generality.
} 
It is now easy to see that the zero mode profiles have the shape given in Plot (c). Moreover, the left-handed zero mode is exponentially localized on $y=0$ and the right-handed one on $y=l$, that is we have

$$
\frac{f_{L}^{(0)}(y=l)}{f_{L}^{(0)}(y=0)}=\frac{f_{R}^{(0)}(y=0)}{f_{R}^{(0)}(y=l)}=e^{-h l} .
$$

Eqs. (2.9) tell us that the bigger is the dimensionless parameter $h l$ the stronger is this localization mechanism.

As we pointed out at the beginning of this section, we now require the lightest wave function $f_{0}(y)$ coming from $A_{\mu}(x, y)$ to depend on some order parameter $v$ for the breaking of the 5D gauge symmetry. Moreover, we require $f_{0}(y)$ to be peaked on $y=0$ for $v \neq 0$ and to correctly reduce to a constant in the unbroken limit $(v \rightarrow 0)$. For example we could choose

$$
f_{0}(y)=N_{0} e^{-a^{2}(v) y^{2}}
$$

with $a(v) \rightarrow 0$ in the unbroken limit. Wave function (2.10) is obviously of the form presented in Plot $(a)$. Now it is already clear that we have a chiral asymmetry in the low energy theory for $v \neq 0$. In order to discuss quantitatively this mechanism we give the couplings between the light $4 \mathrm{D}$ fermions and the light $4 \mathrm{D}$ vector field:

$$
g_{L}=e_{f} \frac{\int d y\left|f_{L}^{(0)}(y)\right|^{2} f_{0}(y)}{\int d y\left|f_{L}^{(0)}(y)\right|^{2}}, \quad g_{R}=e_{f} \frac{\int d y\left|f_{R}^{(0)}(y)\right|^{2} f_{0}(y)}{\int d y\left|f_{R}^{(0)}(y)\right|^{2}}
$$

where the integrals are performed over the complete range of $y$ (in this case $-l \leq y \leq l$ ). Therefore, for $v=0$, we have an exact vector-like spectrum $\left(g_{L}=g_{R}\right)$. But, in the case $v \neq 0$, we have a big chiral asymmetry $g_{L} \gg g_{R}$ when the distance $l$ between the left-handed and the right-handed branes is large, for fixed $h$ and $f_{0}$.

\subsection{Infinite fifth dimension}

In the last subsection we have considered a compact extra dimension. Here we show that an equivalent result can be obtained in the presence of an infinite fifth dimension $(-\infty \leq y \leq+\infty)$. Indeed, since the fermion localization is achieved by the field $\varphi$ and the remaining 4 dimensions are of course assumed to be infinite, it is natural to have also an infinite fifth dimension. Moreover, the set up that we present here provides a non vanishing mass for the lightest fermions.

In this subsection we consider the following configuration for $\varphi$ :

$$
\varphi(y)=h \theta(y)[1-\theta(y-l)]-m,
$$

where $h$ and $m$ are positive constants with dimension of mass and $l$ is again a length scale. Eq. (2.12), which corresponds to the first plot of Figure 1, represents the simplest example of a two domain wall system.

\footnotetext{
${ }^{7}$ Without loss of generality we have also assumed $\int d y\left|f_{0}(y)\right|^{2}=1$.
} 




Figure 1: The first plot represents the two domain wall configuration defined by (2.12). The second plot shows the corresponding profiles of the left-handed (continuous line) and the right-handed (dashed line) lightest normalisable modes, given in Eq. (2.15).

By using (2.6) we obtain the following solutions to the zero mode equations:

$$
\begin{array}{ll}
f_{L}^{(0)}(y) \propto & \exp [-\theta(y) h y+h \theta(y-l)(y-l)+m y], \\
f_{R}^{(0)}(y) \propto & \exp [+\theta(y) h y-h \theta(y-l)(y-l)-m y] .
\end{array}
$$

These functions are both non normalizable and therefore they decouple from the interactive sector of the effective theory. This means that the lightest non-trivial mode is a couple of massive (Weyl) fermions. Here we want to find explicitly their corresponding mass and wave functions and so we consider the Schroedinger eqs. (2.7) for the massive modes: in this case the potentials for the two chiralities are

$$
\begin{aligned}
V_{L, R}= & \left(h^{2}-2 h m\right) \theta(y)[1-\theta(y-l)]+m^{2} \\
& \mp h[\delta(y)-\delta(y-l)],
\end{aligned}
$$

where $\delta$ is the Dirac delta function which emerges from the derivative of the step function $\theta$. We observe that the smooth parts in $V_{L}$ and $V_{R}$ are equal. However, the left-handed modes are subjected to a delta function with a negative coefficient at $y=0$ and with a positive one at $y=l$, whereas, for the right-handed modes, the delta functions are interchanged. Therefore, we expect the lightest (massive) mode to be made of a left-handed mode localized on $y=0$ and a right-handed mode localized on $y=l$. To improve this localization mechanism we also impose $h^{2}-2 h m>0$, which, because of $h>0$, implies $h-m>0$.

By analyzing the Schroedinger equation for $f_{L}$ with standard quantum mechanics methods, 
we find the following bounded solution:

$$
f_{L}=\left\{\begin{array}{c}
A_{L} \exp (q y) \quad \text { for } \quad y<0, \\
\frac{1}{2} A_{L}\left[\left(1+\frac{q-h}{k}\right) \exp (k y)+\left(1+\frac{h-q}{k}\right) \exp (-k y)\right] \quad \text { for } \quad 0 \leq y \leq l, \\
\frac{1}{2} A_{L}\left[\left(1+\frac{q-h}{k}\right) \exp (k l)+\left(1+\frac{h-q}{k}\right) \exp (-k l)\right] \exp (q l-q y) \quad \text { for } \quad y>0,
\end{array}\right.
$$

where $q$ and $k$ are two positive 8 constants defined by

$$
q^{2} \equiv m^{2}-M^{2}, \quad k^{2} \equiv(h-m)^{2}-M^{2},
$$

and $A_{L}$ is a normalisation constant. Moreover, $M$ represents the mass of this solution, which should be computed by solving the following algebraic equation:

$$
\tanh (k l)=\frac{q k}{h m-q^{2}}
$$

The corresponding solution for $f_{R}$ is given by $f_{R}(y) \propto f_{L}(l-y)$ as the potentials satisfy $V_{R}(y)=V_{L}(l-y)$.

We now want to compute explicitly $M$ and show that, for large values of $l, f_{L}$ is localized on $y=0$ and $f_{R}$ is localized on $y=l$. To this end we observe that $\tanh (k l)$ goes to 1 with exponential velocity when $l \rightarrow \infty$ and, by plugging this result into (2.17), we obtain $M \rightarrow 0$. Therefore, we try a solution of the form $M^{2}=M_{0}^{2} \exp (-\alpha l)$, where $M_{0}$ and $\alpha$ are some positive constants, namely a solution that goes exponentially to zero when $l \rightarrow \infty$. By using this ansatz in Eq. (2.17), we obtain

$$
M^{2}=4 m^{2}\left(1-\frac{m}{h}\right)^{2} \exp [-2(h-m) l]+\ldots,
$$

where the dots are small corrections of the order $\exp [-4(h-m) l]$. Therefore, if $l$ is large, we can take just the first term in Eq. (2.18). Since we have an explicit expression for $M$, we can now study the profile of $f_{L, R}$ by plugging (2.18) into (2.15). The result is that $f_{L}$ is exponentially localized on $y=0$ and $f_{R}$ is exponentially localized on $y=l$. In fact we have

$$
\frac{f_{L}(y=l)}{f_{L}(y=0)}=\frac{f_{R}(y=0)}{f_{R}(y=l)}=\left(1-\frac{m}{h}\right) e^{-(h-m) l}+\ldots
$$

where the dots now represent terms of the order $\exp [-2(h-m) l]$. We provide the shape of $f_{L}$ and $f_{R}$ in the second plot of Figure 1 .

We could proceed as in Subsection 2.2 and prove that a big chiral asymmetry emerges in this case if $l$ is large and if the gauge field $A_{\mu}$ satisfies the hypothesis that we mentioned in Subsection 2.2. In the next section we shall discuss how these properties of the gauge field can be generated in a dynamical way.

\footnotetext{
${ }^{8}$ We observe that $q>0$ is a necessary condition for normalisability.
} 


\section{A Bosonic Completion: 5D warped Higgs Model}

In the last section we assumed $A_{M}$ to have the following properties:

1. The spectrum of the $4 \mathrm{D}$ vector fluctuations is made of a light mode and a tower of heavy KK modes.

2. The light spin-1 mode wave function is peaked on the left-handed brane if and only if the gauge symmetry is spontaneously broken.

The main purpose of the present section is to obtain dynamically these properties in a simple way.

To this end we introduce, apart from a 5D $U(1)$ gauge field $A_{M}$, a charged Higgs field $\phi$ with a Mexican hat potential and we assume the following bosonic action?:

$$
S_{B}=\int d^{5} X\left\{-\frac{\Delta}{4} F_{M N} F^{M N}-\Delta_{S}\left[\left(D_{M} \phi\right)^{\dagger} D^{M} \phi+V(\phi)\right]\right\}
$$

where $F_{M N}=\partial_{M} A_{N}-\partial_{N} A_{M}, D_{M} \phi \equiv\left(\partial_{M}+i e A_{M}\right) \phi, V(\phi) \equiv \lambda\left(|\phi|^{2}-v^{2}\right)^{2}, e$ and $v$ are real constants, $\lambda$ is a positive constant and the weight functions $\Delta$ and $\Delta_{S}$ depend only on $y$. The $U(1)$ gauge symmetry acts on $A_{M}$ and $\phi$ as follows

$$
A_{M} \rightarrow A_{M}-\partial_{M} \alpha, \quad \phi \rightarrow e^{i e \alpha} \phi .
$$

We have introduced the $\Delta$-weights in order to have Property 1 . Indeed, as we have seen in Section 2, the distance $l$ between the left-handed and right-handed branes, and therefore the size of the fifth dimension, should be large in order to achieve a big chiral asymmetry. In this limit, the KK mass scale becomes very small in standard KK scenarios, where one does not introduce any weight function and assumes a compact internal space. Instead, by choosing $\Delta$ and $\Delta_{S}$ in a suitable way, we can decouple the KK mass gap and the volume of the internal space 10 . In the following we shall take an infinite extra dimension $(-\infty<y<+\infty)$ as the localization of the spin-1 and spin-0 fields can be obtained by means of the weight function 11 . Here we do not describe the origin of the weight functions, but we observe that they may arise for example from warped solutions of field theories that include gravity [5, 6]. For this reason we shall refer to the model defined by $S_{B}$ as a $5 \mathrm{D}$ warped Higgs model.

The EOMs, which correspond to $S_{B}$, are

$$
\begin{aligned}
& \frac{1}{\Delta} \partial_{M}\left(\Delta F^{M N}\right)=i e \frac{\Delta_{S}}{\Delta}\left[\left(D^{N} \phi\right)^{\dagger} \phi-\phi^{\dagger} D^{N} \phi\right], \\
& \frac{1}{\Delta_{S}} D_{M}\left(\Delta_{S} D^{M} \phi\right)=\frac{\partial V}{\partial \phi^{\dagger}} .
\end{aligned}
$$

In the rest of the paper we assume the following VEV:

$$
\left\langle A_{M}\right\rangle=0, \quad\langle\phi\rangle=v,
$$

\footnotetext{
${ }^{9}$ We choose signature $(-1,+1,+1,+1,+1)$.

${ }^{10}$ Recently it has been shown that, in the presence of warping, such a decoupling may happen even if one takes into account the gravitational backreaction 13 .

${ }^{11}$ This set up is not necessary and we could consider a compact internal space.
} 
which is a solution to the EOMs and represents the simplest way to realize the SSB of the gauge symmetry.

\subsection{Gauge fixing and perturbations}

We now want to study in details the linear perturbations around the VEV (3.23). To this end we first have to consistently fix a gauge in order to focus only on the physical spectrum coming from $A_{M}$ and $\phi=v+(\sigma+i \eta) / \sqrt{2}$. Therefore, we have to add a gauge fixing term $\mathcal{L}_{G F}$ to the $5 \mathrm{D}$ lagrangian. We choose 12

$$
\mathcal{L}_{G F}=-\frac{\Delta}{2}\left[\frac{1}{\Delta} \partial_{M}\left(\Delta A^{M}\right)+\sqrt{2} e v \frac{\Delta_{S}}{\Delta} \eta\right]^{2}
$$

because, as we show below, in this gauge the spin- 1 and spin-0 fluctuations do not mix at the bilinear level in the action. This gauge is a generalization of the $R_{\xi}$ gauge (with $\xi=1$ ) to warped $5 \mathrm{D}$ model 13 . In the appendix we demonstrate, with a perturbative argument, that (3.24) is a legitimate gauge fixing term.

The complete bosonic action $S_{B}^{\prime}=S_{B}+S_{G F}$, where $S_{G F}=\int d^{5} X \mathcal{L}_{G F}$, can be written in terms of the fluctuations $A_{M}, \sigma$ and $\eta$ as follows:

$$
\begin{aligned}
S_{B}^{\prime}= & \int d^{5} X\left\{-\frac{\Delta}{2} \partial_{M} A_{N} \partial^{M} A^{N}+\frac{\Delta}{2} \partial_{y}^{2} \ln \Delta A_{y}^{2}-e^{2} v^{2} \Delta_{S} A_{M} A^{M}-\frac{\Delta_{S}}{2} \partial_{M} \sigma \partial^{M} \sigma\right. \\
& -\frac{\Delta_{S}}{2} \partial_{M} \eta \partial^{M} \eta-2 \lambda v^{2} \Delta_{S} \sigma^{2}-e^{2} v^{2} \frac{\Delta_{S}^{2}}{\Delta} \eta^{2}+\sqrt{2} e v \Delta \partial_{y}\left(\frac{\Delta_{S}}{\Delta}\right) A_{y} \eta \\
& +e \Delta_{S} A_{M}\left(\eta \partial^{M} \sigma-\sigma \partial^{M} \eta\right)-\sqrt{2} v e^{2} \Delta_{S} A_{M} A^{M} \sigma-\frac{e^{2}}{2} \Delta_{S} A_{M} A^{M}\left(\sigma^{2}+\eta^{2}\right) \\
& \left.-\sqrt{2} v \lambda \Delta_{S} \sigma\left(\sigma^{2}+\eta^{2}\right)-\frac{\lambda}{4} \Delta_{S}\left(\sigma^{2}+\eta^{2}\right)^{2}\right\}
\end{aligned}
$$

where we have neglected boundary terms, that is all the terms of the form $\int d^{5} X \partial_{M} F$, with $F$ a functional of the fields, and we have used that $\Delta$ and $\Delta_{S}$ depend only on $y$. The first two lines in (3.25) represent the bilinear terms in the bosonic sector, whereas the third and fourth lines are the interaction terms. It is now clear that the spin- 1 field $A_{\mu}$ and the spin-0 fields $A_{y}, \sigma$ and $\eta$ do not mix at the bilinear level. However, we have a non-trivial mixing between $A_{y}$ and $\eta$. We shall solve this problem in Subsection 3.3.

From the 5D point of view, $\eta$ represents the would-be Goldstone boson for the breaking of the U(1) gauge symmetry. This field appears explicitly in the lagrangian because our gauge (3.24) is not the unitary gauge, which instead corresponds to the $R_{\xi}$ gaug 14 with $\xi \rightarrow \infty$.

\footnotetext{
${ }^{12}$ We observe that this gauge fixing leads to a non-trivial ghost action. However, we do not analyze such a term because, in the present paper, we only compute some observable quantities in the semiclassical approximation, where there are no ghost contributions.

${ }^{13}$ For a discussion on some generalizations of the $R_{\xi}$ gauge in unwarped models see [14].

${ }^{14}$ See the appendix for the definition of the $R_{\xi}$ gauges in the presence of warping.
} 


\section{$3.2 \quad$ Spin-1 sector and coupling with fermions}

In this subsection we focus on the spin- 1 sector, which is crucial in our discussion on the chiral asymmetry and the Higgs mechanism, and we study the linearized EOMs for this sector.

To this end we need the bilinear action for $A_{\mu}$, which, thanks to (3.25), is simply

$$
S_{2}\left(A_{\mu}\right)=\int d^{5} X\left(-\frac{\Delta}{2} \partial_{N} A_{\mu} \partial^{N} A^{\mu}-e^{2} v^{2} \Delta_{S} A_{\mu} A^{\mu}\right)
$$

If we take the variation of $S_{2}\left(A_{\mu}\right)$ under $A_{\mu} \rightarrow A_{\mu}+\delta A_{\mu}$ we obtain the following linearized EOMs:

$$
\frac{1}{\Delta} \partial_{M}\left(\Delta \partial^{M} A^{\mu}\right)=2 e^{2} v^{2} \frac{\Delta_{S}}{\Delta} A^{\mu}
$$

where, as we did in the derivation of (3.25), we have neglected boundary terms. In the case of $A_{\mu}$, this constraint can be written as follows [15, 13]:

$$
\int d y \partial_{y}\left(\Delta \delta A_{\mu} \partial_{y} A^{\mu}\right)=0
$$

Eq. (3.28) represents a boundary condition for the wave functions of the spin-1 fields along the extra dimension.

In order to analyze the $4 \mathrm{D}$ spectrum we perform a $\mathrm{KK}$ expansion,

$$
A_{\mu}(x, y)=\sum_{n} A_{\mu}^{(n)}(x) f_{n}(y)
$$

and a Fourier expansion of the 4D fields: $A_{\mu}^{(n)}(x) \propto e^{-i p_{n} x}$. Eq. (3.27) now becomes

$$
-\frac{1}{\Delta} \partial_{y}\left(\Delta \partial_{y} f_{n}\right)+2 e^{2} v^{2} \frac{\Delta S}{\Delta} f_{n}=M_{n}^{2} f_{n}
$$

where $M_{n}^{2}=-p_{n}^{2}$. The solutions to the latter equation, which satisfy the boundary condition (3.28), represent the physical spin-1 sector that we are interested in. Eq. (3.29) can be transformed in the standard Schroedinger form by means of the definition $\chi_{n} \equiv \Delta^{1 / 2} f_{n}$ :

$$
\left[-\partial_{y}^{2}+\mathcal{V}\right] \chi_{n}=M_{n}^{2} \chi_{n}
$$

where the potential $\mathcal{V}$ turns out to be

$$
\mathcal{V}=\frac{1}{4}\left(\partial_{y} \ln \Delta\right)^{2}+\frac{1}{2} \partial_{y}^{2} \ln \Delta+2 e^{2} v^{2} \frac{\Delta_{S}}{\Delta}
$$

By expressing (3.28) in terms of $\chi_{n}$, we also obtain

$$
\int d y \partial_{y}\left(\chi_{n^{\prime}} \partial_{y} \chi_{n}-\frac{1}{2} \partial_{y} \ln \Delta \chi_{n^{\prime}} \chi_{n}\right)=0, \quad \forall n, n^{\prime} .
$$

This boundary condition implies the hermiticity of the hamiltonian in the Schroedinger problem, and, therefore, we shall call it hermiticity condition (HC) [13]. 
So far we have considered general values of $\Delta$ and $\Delta_{S}$. In the rest of this subsection we discuss the particular choice

$$
\Delta(y)=\exp \left(-\frac{1}{2} \mathcal{M}^{2} y^{2}\right), \quad \Delta_{S}(y)=\frac{\delta^{2}}{8} y^{2} \exp \left(-\frac{1}{2} \mathcal{M}^{2} y^{2}\right),
$$

where $\mathcal{M}$ and $\delta$ are positive constants with the dimension of mass. Indeed, as we prove below, (3.32) leads to Properties 1 and 2 in a very simple way 15 . By plugging (3.32) into (3.30) we find

$$
\mathcal{V}(y)=\frac{1}{4} \mathcal{M}^{4}\left(1+\epsilon^{2}\right) y^{2}-\frac{1}{2} \mathcal{M}^{2}
$$

where

$$
\epsilon^{2} \equiv e^{2} v^{2} \delta^{2} / \mathcal{M}^{4}
$$

Therefore, we have a harmonic oscillator potential. We observe that the only effect of the SSB $(v \neq 0)$ is to change the "frequency" of the harmonic oscillator as follows: $\mathcal{M}^{2} \rightarrow \mathcal{M}^{2} \sqrt{1+\epsilon^{2}} \equiv$ $\mathcal{M}_{T}^{2}$. It is now trivial to obtain the spectrum, which is given by the harmonic oscillator wave functions and "energy" eigenvalues:

$$
\begin{aligned}
\chi_{n}(y) & =N_{n}\left(y-\frac{2}{\mathcal{M}_{T}^{2}} \partial_{y}\right)^{n} \exp \left(-\frac{1}{4} \mathcal{M}_{T}^{2} y^{2}\right), \\
M_{n}^{2} & =\mathcal{M}_{T}^{2}\left(n+\frac{1}{2}\right)-\frac{1}{2} \mathcal{M}^{2}, \quad n=0,1,2, \ldots,
\end{aligned}
$$

where $N_{n}$ are normalization constants that can be fixed by requiring standard kinetic terms for $A_{\mu}^{(n)}$. Moreover, it is easy to see that the solutions given in (3.35) automatically satisfy (3.31).

Therefore, the spectrum has the following features. We have a first light mode with mass squared $M_{0}^{2}=\frac{1}{2}\left(\mathcal{M}_{T}^{2}-\mathcal{M}^{2}\right)$, which vanishes only for $v=0$. The corresponding wave function $f_{0}$ is

$$
f_{0}(y)=N_{0} \exp \left[-\frac{1}{4}\left(\mathcal{M}_{T}^{2}-\mathcal{M}^{2}\right) y^{2}\right] .
$$

Hence, for $v=0$ we have a constant profile corresponding to a massless gauge field, whereas in the broken case $v \neq 0$ the lightest spin- 1 field acquires a mass and it is localized on $y=0$ by means of a gaussian distribution of the form (2.10). The remaining spin-1 states have very large masses if $\mathcal{M}_{T}^{2}-\mathcal{M}^{2} \ll \mathcal{M}^{2}$, which requires $\epsilon^{2} \ll 1$. In this limit $\epsilon^{2}$ is of the same order of magnitude as the ratio between the squared mass of the light spin-1 field and the KK squared mass scale $\mathcal{M}^{2}$ :

$$
\epsilon^{2} \sim \frac{M_{0}^{2}}{\mathcal{M}^{2}}
$$

So we find that Properties 1 and 2 are satisfied and, therefore, (3.32) is a good choice to realize the mechanism that we discussed in Section 2 ,

\footnotetext{
${ }^{15}$ Of course we do not expect (3.32) to be the only set up that leads to those properties, but we assume (3.32) for the sake of definiteness.
} 
Finally we note that, in order to obtain a large chiral asymmetry in the broken phase, we need $\left(\mathcal{M}_{T}^{2}-\mathcal{M}^{2}\right) l^{2} \gg 1$, where $l$ is the distance between the left-handed and the right-handed branes, which we have introduced in Section 2, Therefore, by using $\epsilon^{2} \ll 1$, we find $\mathcal{M}^{2} \gg l^{-2}$, namely that a decoupling between the KK mass scale and the size of the extra dimension is needed, as we discussed at the beginning of this section.

\subsection{Spin-0 sector}

We now complete the study of the linear perturbations around (3.23) by analyzing the spin-0 sector. Indeed, this is necessary in order to know the complete low energy field content and if a large mass gap between the light and heavy modes emerges in all sectors.

We first examine the spectrum coming from $\sigma$, the 5D physical Higgs field. By using a method similar to what we have applied in the spin-1 sector, we obtain the following linearized EOM and HC:

$$
\frac{1}{\Delta_{S}} \partial_{M}\left(\Delta_{S} \partial^{M} \sigma\right)=4 \lambda v^{2} \sigma, \quad \int d y \partial_{y}\left(\Delta_{S} \delta \sigma \partial_{y} \sigma\right)=0
$$

After performing a KK decomposition $\sigma(x, y)=\sum_{n} \sigma_{n}(x) f_{\sigma n}(y)$ and a Fourier expansion of the $4 \mathrm{D}$ fields $\sigma_{n}(x) \propto e^{-i p_{n} x}$, again we obtain a Schroedinger equation

$$
\left[-\partial_{y}^{2}+\mathcal{V}_{\sigma}\right] \chi_{\sigma n}=M_{n}^{2} \chi_{\sigma n}, \quad \mathcal{V}_{\sigma}=\frac{1}{4}\left(\partial_{y} \ln \Delta_{S}\right)^{2}+\frac{1}{2} \partial_{y}^{2} \ln \Delta_{S}+4 \lambda v^{2},
$$

where $\chi_{\sigma n} \equiv \Delta_{S}^{1 / 2} f_{\sigma n}$, and the following expression for the HC:

$$
\int d y \partial_{y}\left(\chi_{\sigma n^{\prime}} \partial_{y} \chi_{\sigma n}-\frac{1}{2} \partial_{y} \ln \Delta_{S} \chi_{\sigma n^{\prime}} \chi_{\sigma n}\right)=0, \quad \forall n, n^{\prime} .
$$

If we consider Set up (3.32), the potential for $\sigma$ turns out to be

$$
\mathcal{V}_{\sigma}=\frac{1}{4} \mathcal{M}^{4} y^{2}-\frac{3}{2} \mathcal{M}^{2}+4 \lambda v^{2}
$$

which is again a harmonic oscillator potential. Therefore, we find the following wave functions and masses squared:

$$
\begin{aligned}
\chi_{\sigma n}(y) & =N_{\sigma n}\left(y-\frac{2}{\mathcal{M}^{2}} \partial_{y}\right)^{n} \exp \left(-\frac{1}{4} \mathcal{M}^{2} y^{2}\right) \\
M_{\sigma n}^{2} & =\mathcal{M}^{2}\left(n+\frac{1}{2}\right)-\frac{3}{2} \mathcal{M}^{2}+4 \lambda v^{2}, \quad n=1,3,5, \ldots
\end{aligned}
$$

The wave functions with $n$ even do not appear in (3.42) because they do not satisfy the HC (3.40). To illustrate this point let us take $n=0$ and $n^{\prime}=0$ in the left hand side of (3.40): we obtain

$$
\int d y \partial_{y}\left(\chi_{\sigma 0} \partial_{y} \chi_{\sigma 0}+\frac{1}{2} \mathcal{M}^{2} y \chi_{\sigma 0}^{2}-\frac{1}{y} \chi_{\sigma 0}^{2}\right) ;
$$

the first two terms in the previous expression vanish but the third one does not because of the $1 / y$ singularity, which is not canceled by $\chi_{\sigma 0}^{2}$. Indeed, this result is not restricted to the $n=0$ 
wave function, but it holds for all the wave functions with $n$ even, as they are all non-vanishing at $y=0$. Hence the set of fluctuations emerging from $\sigma$ contains a light mode that corresponds to $n=1$ in (3.42) and a tower of KK modes. The latter modes are much heavier than the $n=1$ mode when $\lambda v^{2} \ll \mathcal{M}^{2}$.

To complete the spin-0 sector we now have to examine the fluctuations $A_{y}$ and $\eta$, which are coupled even at the bilinear level. Indeed, the linearized EOMs for these fields are

$$
\begin{aligned}
\frac{1}{\Delta} \partial_{M}\left(\Delta \partial^{M} A_{y}\right) & =-\partial_{y}^{2} \ln \Delta A_{y}+2 e^{2} v^{2} \frac{\Delta_{S}}{\Delta} A_{y}-\sqrt{2} e v \partial_{y}\left(\frac{\Delta_{S}}{\Delta}\right) \eta, \\
\frac{1}{\Delta_{S}} \partial_{M}\left(\Delta_{S} \partial^{M} \eta\right) & =2 e^{2} v^{2} \frac{\Delta_{S}}{\Delta} \eta-\sqrt{2} e v \partial_{y} \ln \frac{\Delta_{S}}{\Delta} A_{y}
\end{aligned}
$$

and the corresponding $\mathrm{HCs}$ are

$$
\int d y \partial_{y}\left(\Delta \delta A_{y} \partial_{y} A_{y}\right)=0, \quad \int d y \partial_{y}\left(\Delta_{S} \delta \eta \partial_{y} \eta\right)=0
$$

We observe that Eqs. (3.43) can be written in a Schroedinger form by means of the transformations $\tilde{A}_{y} \equiv \Delta^{1 / 2} A_{y}$ and $\tilde{\eta} \equiv \Delta_{S}^{1 / 2} \eta$ :

$$
\begin{aligned}
-\partial_{y}^{2} \tilde{A}_{y}+\mathcal{V}_{y} \tilde{A}_{y}+\mathcal{C} \tilde{\eta} & =\partial_{\mu} \partial^{\mu} \tilde{A}_{y} \\
-\partial_{y}^{2} \tilde{\eta}+\mathcal{V}_{\eta} \tilde{\eta}+\mathcal{C} \tilde{A}_{y} & =\partial_{\mu} \partial^{\mu} \tilde{\eta}
\end{aligned}
$$

where

$$
\begin{aligned}
\mathcal{V}_{y} & =\frac{1}{4}\left(\partial_{y} \ln \Delta\right)^{2}-\frac{1}{2} \partial_{y}^{2} \ln \Delta+2 e^{2} v^{2} \frac{\Delta_{S}}{\Delta} \\
\mathcal{V}_{\eta} & =\frac{1}{4}\left(\partial_{y} \ln \Delta_{S}\right)^{2}+\frac{1}{2} \partial_{y}^{2} \ln \Delta_{S}+2 e^{2} v^{2} \frac{\Delta_{S}}{\Delta} \\
\mathcal{C} & =-\sqrt{2} e v\left(\frac{\Delta}{\Delta_{S}}\right)^{1 / 2} \partial_{y}\left(\frac{\Delta_{S}}{\Delta}\right) .
\end{aligned}
$$

We observe that Eqs. (3.45) are also coupled and it is in general difficult to find a complete set of solutions for general $\Delta$ and $\Delta_{S}$. However, this problem can be easily solved if we consider the special case given in (3.32). In fact, by using (3.32) we obtain

$$
\mathcal{V}_{\eta}=\frac{1}{4} \mathcal{M}_{T}^{4} y^{2}-\frac{3}{2} \mathcal{M}^{2}, \quad \mathcal{V}_{y}=\mathcal{V}_{\eta}+2 \mathcal{M}^{2}, \quad \mathcal{C}=-e v \delta
$$

So $\mathcal{V}_{\eta}$ and $\mathcal{V}_{y}$ are two harmonic oscillator potential and $\mathcal{C}$ is constant. These properties allow us to easily decouple System (3.45). The mass eigenstates can be expressed as follows:

$$
\left(\begin{array}{l}
\xi_{1} \\
\xi_{2}
\end{array}\right) \equiv\left(\begin{array}{cc}
\cos \theta & \sin \theta \\
-\sin \theta & \cos \theta
\end{array}\right)\left(\begin{array}{c}
\tilde{A}_{y} \\
\tilde{\eta}
\end{array}\right)
$$

where the mixing angle $\theta$ is defined by

$$
\cos ^{2} \theta \equiv \frac{\epsilon^{2}}{\epsilon^{2}+\left(\sqrt{1+\epsilon^{2}}-1\right)^{2}} .
$$


We recall that the parameter $\epsilon$, which we have defined in (3.34), is very small because of (3.37). This implies that $\theta$ is very small too: $\cos ^{2} \theta=1-\epsilon^{2} / 4+O\left(\epsilon^{4}\right)$. If we plug (3.48) into (3.45), we find two decoupled Schroedinger equations with potentials $\mathcal{V}_{1,2}=\frac{1}{4} \mathcal{M}_{T}^{4} y^{2}-\frac{1}{2} \mathcal{M}^{2} \pm \mathcal{M}_{T}^{2}$. The wave functions and masses squared associated to $\xi_{i}, i=1,2$, are

$$
\begin{aligned}
& \chi_{i, n}(y)=N_{i, n}\left(y-\frac{2}{\mathcal{M}_{T}^{2}} \partial_{y}\right)^{n} \exp \left(-\frac{1}{4} \mathcal{M}_{T}^{2} y^{2}\right) \\
& M_{1(2), n}^{2}=\mathcal{M}_{T}^{2}\left(n+\frac{1}{2} \pm 1\right)-\frac{1}{2} \mathcal{M}^{2}, \quad n=1,3,5, \ldots
\end{aligned}
$$

where again we have performed a KK decomposition $\xi_{i}(x, y)=\sum_{n} \xi_{i, n}(x) \chi_{i n}(y)$ and a $4 \mathrm{D}$ Fourier expansion $\xi_{i, n}(x) \propto e^{-i p_{i n} x}$. We observe that, as in (3.42), we do not have the even values of $n$ in the harmonic oscillator spectrum because of the HCs. In fact, if we plug (3.48) and the definitions $\tilde{A}_{y} \equiv \Delta^{1 / 2} A_{y}$ and $\tilde{\eta} \equiv \Delta_{S}^{1 / 2} \eta$ into (3.44) we find

$$
\begin{aligned}
\int d y \partial_{y}\left[\left(\cos \theta \delta \xi_{1}-\sin \theta \delta \xi_{2}\right)\left(\partial_{y}-\frac{1}{2} \partial_{y} \ln \Delta\right)\left(\cos \theta \xi_{1}-\sin \theta \xi_{2}\right)\right] & =0 \\
\int d y \partial_{y}\left[\left(\sin \theta \delta \xi_{1}+\cos \theta \delta \xi_{2}\right)\left(\partial_{y}-\frac{1}{2} \partial_{y} \ln \Delta_{S}\right)\left(\sin \theta \xi_{1}+\cos \theta \xi_{2}\right)\right] & =0
\end{aligned}
$$

We now note that in (3.51) we have $\partial_{y} \ln \Delta$, whereas in (3.52) we have $\partial_{y} \ln \Delta_{S}$. Therefore, as we discussed below Eq. (3.42), Condition (3.51) is weaker than (3.52) and we can just focus on the latter. Also, if we first set $\xi_{2}=0$ and $\delta \xi_{2}=0$ and we keep $\xi_{1}$ and $\delta \xi_{1}$ non vanishing and then we exchang 16 the role of $\left\{\xi_{1}, \delta \xi_{1}\right\}$ and $\left\{\xi_{2}, \delta \xi_{2}\right\}$, Condition (3.52) implies

$$
\int d y \partial_{y}\left[\delta \xi_{i}\left(\partial_{y}-\frac{1}{2} \partial_{y} \ln \Delta_{S}\right) \xi_{i}\right]=0
$$

We can now apply the argument given below Eq. (3.42) and find that only the odd values of $n$ are not projected out by the HCs.

So we have found that the sector $\left\{A_{y}, \eta\right\}$ contains just one light mode $\left(\xi_{2,1}, i=2, n=1\right)$ and a tower of heavy modes with masses at least of the order $\mathcal{M}$.

\subsection{Counting the degrees of freedom}

We conclude this section by summarizing the spectrum that we have found in the case of a gaussian form (3.32) for the weight functions. All the Schroedinger equations, which determine the mass spectrum and the profiles along the extra dimension, turn out to have a harmonic oscillator potential. However, the "frequencies" of the oscillators and the boundary conditions are different in various sectors. We summarize our results in Table 1.

We observe that the low energy spectrum $(E \ll \mathcal{M})$ contains a vector field with squared mass $\left(\mathcal{M}_{T}^{2}-\mathcal{M}^{2}\right) / 2$ and only two scalar fields with squared masses $4 \lambda v^{2}$ and $\left(\mathcal{M}_{T}^{2}-\mathcal{M}^{2}\right) / 2$. Indeed, the field $\xi_{1}$ coming from the sector $\left\{A_{y}, \eta\right\}$ contains only heavy modes. We conclude

\footnotetext{
${ }^{16}$ This is possible because $\xi_{1}, \delta \xi_{1}, \xi_{2}$ and $\delta \xi_{2}$ are all independent.
} 


\begin{tabular}{|l|l|l|}
\hline Sector & "Frequency" & Squared masses \\
\hline$A_{\mu}$ & $\mathcal{M}_{T}^{2}$ & $\mathcal{M}_{T}^{2}\left(n+\frac{1}{2}\right)-\frac{1}{2} \mathcal{M}^{2}, n=0,1,2, \ldots$ \\
$\sigma$ & $\mathcal{M}^{2}$ & $\mathcal{M}^{2}\left(n+\frac{1}{2}\right)-\frac{3}{2} \mathcal{M}^{2}+4 \lambda v^{2}, n=1,3,5 \ldots$ \\
$\left\{A_{y}, \eta\right\}$ & $\mathcal{M}_{T}^{2}$ & $\mathcal{M}_{T}^{2}\left(n+\frac{1}{2} \pm 1\right)-\frac{1}{2} \mathcal{M}^{2}, n=1,3,5 \ldots$ \\
\hline
\end{tabular}

Table 1: The complete bosonic spectrum in Case (3.32). All the effective Schroedinger problems turn out to have a harmonic oscillator potential with frequency given in the second column. The allowed levels in the harmonic oscillator spectrum are determined by the HCs.

that the low energy spectrum and degrees of freedom are the same as in the $4 \mathrm{D}$ spontaneously broken Higgs model in the $R_{1}$ gaug£ 17 .

In the next section we shall use the mass spectrum and the wave functions that we have found to study some interactions in the $4 \mathrm{D}$ effective theory.

\section{4D Effective Theory and Gauge Invariance}

In this section we study the form of the $4 \mathrm{D}$ effective theory for the light bosonic modes. As we will see, when the $5 \mathrm{D}$ gauge symmetry is broken $(v \neq 0)$, the action for such a theory in general cannot be written as a gauge invariant action with at most a SSB of the gauge symmetry. We prove this statement by choosing the weight functions (3.32) and exploiting the exact results of the previous section. However, by using a semiclassical approximation, we also show that the terms which explicitly break the 4D gauge invariance are very small (of the order of $\epsilon^{2}$ ) and, therefore, in the limit in which the $5 \mathrm{D}$ gauge symmetry is restored $(v \rightarrow 0)$ these terms go to zero and the $4 \mathrm{D}$ effective theory acquires an exact gauge symmetry.

As stated in Subsection 3.4, the low energy spectrum is made of a vector boson $V_{\mu} \equiv A_{\mu}^{(0)}$ and two scalar fields $\omega_{1} \equiv \sigma_{1}$ and $\omega_{2} \equiv \xi_{2,1}$, where the mass of $\omega_{2}$ is equal to the mass of $V_{\mu}$. Therefore, if there was a gauge symmetry at low energy, the $4 \mathrm{D}$ effective action would have the following form

$$
\begin{aligned}
\hat{S}+\hat{S}_{G F}=\int d^{4} x[- & \frac{1}{4} V_{\mu \nu} V^{\mu \nu}-\left(D_{\mu} \omega\right)^{\dagger} D^{\mu} \omega-U(|\omega|) \\
& \left.-\frac{1}{2}\left(\partial_{\mu} V^{\mu}+\sqrt{2} \hat{e} \hat{v} \omega_{2}\right)^{2}+\ldots\right]
\end{aligned}
$$

namely it should be the action of a $4 \mathrm{D}$ Higgs model in the $R_{1}$ gauge, apart from higher dimensional operators that we denoted in (4.54) with the dot:18. In (4.54) we have introduced $V_{\mu \nu} \equiv \partial_{\mu} V_{\nu}-\partial_{\nu} V_{\mu}$, the complex field $\omega \equiv \hat{v}+\left(\omega_{1}+i \omega_{2}\right) / \sqrt{2}$, the covariant derivative $D_{\mu} \omega=$ $\left(\partial_{\mu}+i \hat{e} V_{\mu}\right) \omega$ and a general $\mathrm{U}(1)$-invariant potential $U(|\omega|)$. However, in this section we show that in general this is not the case when $v \neq 0$. To this end we explicitly write (4.54) in terms

\footnotetext{
${ }^{17}$ In the $R_{1}$ gauge the mass of the vector boson is equal to the mass of the would-be Goldstone boson.

${ }^{18}$ We note that the $4 \mathrm{D}$ effective theory generally contains non renormalisable interactions and therefore the theorem proved in [16] is not applicable.
} 
of $V_{\mu}, \omega_{1}$ and $\omega_{2}$ :

$$
\begin{array}{r}
\hat{S}+\hat{S}_{G F}=\int d^{4} x\left[-\frac{1}{2} \partial_{\mu} V_{\nu} \partial^{\mu} V^{\nu}-\hat{e}^{2} \hat{v}^{2} V_{\mu} V^{\mu}-\frac{1}{2} \partial_{\mu} \omega_{i} \partial^{\mu} \omega_{i}-\hat{e}^{2} \hat{v}^{2} \omega_{2}^{2}\right. \\
\left.+\hat{e} V_{\mu}\left(\omega_{2} \partial^{\mu} \omega_{1}-\omega_{1} \partial^{\mu} \omega_{2}\right)-\sqrt{2} \hat{e}^{2} \hat{v} \omega_{1} V_{\mu} V^{\mu}-\frac{1}{2} \hat{e}^{2} V_{\mu} V^{\mu}\left(\omega_{1}^{2}+\omega_{2}^{2}\right)+U(|\omega|)+\ldots\right]
\end{array}
$$

where we have taken $\hat{v}$ real without loss of generality. Since $U$ depends only on $|\omega|$ it possibly contributes to the mass term of $\omega_{1}$, but it gives no contribution to the mass term of $\omega_{2}$.

\subsection{Small explicit breaking of gauge invariance}

We now compare the 4D effective theory descending from the 5D theory of the previous section with the $4 \mathrm{D}$ theory defined by (4.55). For simplicity we perform only semiclassical calculation, that is we neglect all loop contributions to the effective theory. Let us first ignore the higher dimensional operators that are represented by the dots in (4.54); afterwards we will show that such operators do not ruin the argument we are going to present here.

If we compare the spectrum found in the previous section with the bilinear terms in (4.55) we obtain that gauge invariance requires

$$
2 \hat{e}^{2} \hat{v}^{2}=\frac{1}{2}\left(\mathcal{M}_{T}^{2}-\mathcal{M}^{2}\right)
$$

This relation fixes one parameter out of $\hat{e}^{2}$ and $\hat{v}^{2}$. The other parameter, say $\hat{e}^{2}$, can be fixed by looking at the cubic operator

$$
\omega_{1} V_{\mu} V^{\mu}
$$

In a realistic model an interaction of this type contributes to the decay of a physical Higgs into two massive vector bosons. After a bit of algebra we obtain

$$
\hat{e}^{2}=\frac{1}{2 \sqrt{2 \pi}} \frac{e^{2} \mathcal{M} \epsilon^{2}}{\left(1+\epsilon^{2}\right)\left(\sqrt{1+\epsilon^{2}}-1\right)} .
$$

We can now test the gauge invariance of the $4 \mathrm{D}$ effective theory by examining the operator

$$
V_{\mu} V^{\mu} \omega_{1}^{2}
$$

If we denote with $-g^{2} / 2$ the corresponding coupling constant, from (4.55) we have that gauge invariance requires

$$
g^{2}=\hat{e}^{2} .
$$

In the following we show that Relation (4.60) is not exactly satisfied, but it is broken by very small contributions of the order of $\epsilon^{2}$. To prove this statement we observe that the operator that we are considering has dimension 4 and it may have both light mode contributions and heavy KK mode contributions [7, 17.

We first analyze the light mode contribution $g_{l m}^{2}$ to $g^{2}$, which can be obtained by considering the term $-e^{2} \Delta_{S} A_{\mu} A^{\mu} \sigma^{2} / 2$ in the third line of (3.25), neglecting the heavy KK modes and 


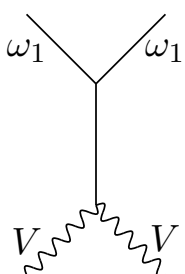

(a)

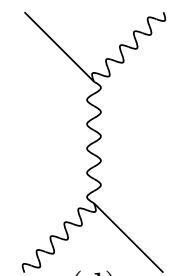

(d)

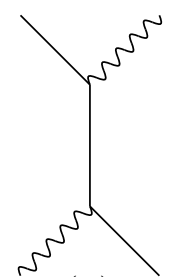

(b)

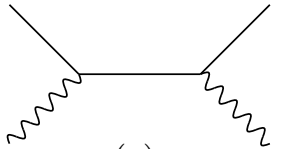

(c)

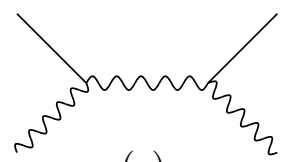

(e)

Figure 2: The only types of tree diagrams representing the heavy mode contribution to the scattering $V, \omega_{1} \rightarrow$ $V, \omega_{1}$. Straight lines are associated to scalar particles, whereas wavy lines to vector particles. The internal lines are heavy mode propagators.

integrating over the extra dimension. Thanks to our exact results of Section 3 we can explicitly compute this contribution and we find

$$
g_{l m}^{2}=\frac{1}{\sqrt{2 \pi}} \frac{e^{2} \mathcal{M}}{\sqrt{1+\epsilon^{2}}} .
$$

We now pass to the heavy mode contribution to (4.59) and in order to determine it we consider the scattering $V, \omega_{1} \rightarrow V, \omega_{1}$, which represents the scattering between the Higgs field and the light massive vector. By looking at Action (3.25) and by using the KK expansions, we find five types of heavy mode contributions (see Figure 2). Diagrams of Type (a) do not contribute as the vertex with two $\omega_{1}$-lines and one heavy scalar is proportional to $\int d y \Delta_{S}^{-1 / 2} \chi_{\sigma 1}^{2} \chi_{\sigma n} \propto \int d y \chi_{\sigma 1} \chi_{\sigma n}=0$, where we used $\Delta_{S} \propto \chi_{\sigma 1}^{2}$ and $n=3,5,7 \ldots$ Diagrams (b) and (c) are negligible as they lead to higher dimensional operators in the effective theory, which involve derivatives. Indeed, one can show that those diagrams contribute only at the order $\epsilon^{4}$ to $g$, if one consistently requires that the momenta of the internal propagators are much smaller than $\mathcal{M}$. Finally, we consider Diagrams (d) and (e), which involve a cubic interaction between two vector bosons and one $\omega_{1}$. This interaction is given by the term $-\sqrt{2} v e^{2} \Delta_{S} A_{\mu} A^{\mu} \sigma$ in the third line of (3.25). Since this term is proportional to $v$, Diagrams (d) and (e) do not contribute to $g$ at the order 1 , but only at the order $\epsilon^{2}$ : we find

$$
g_{h m}^{2}=-\frac{1}{\sqrt{2 \pi}} \frac{e^{2} \mathcal{M} \epsilon^{2}\left(1+\epsilon^{2}\right)^{-1}}{\sqrt{1+\epsilon^{2}}+\frac{1}{4} \sqrt{1+\epsilon^{2}}-\frac{1}{4}},
$$

where $g_{h m}^{2}$ is the heavy mode contribution to $g^{2}$. We can now write

$$
g^{2}-\hat{e}^{2}=g_{l m}^{2}+g_{h m}^{2}-\hat{e}^{2}=-\frac{3}{4} \frac{e^{2} \mathcal{M}}{\sqrt{2 \pi}} \epsilon^{2}+O\left(\epsilon^{4}\right) .
$$

The latter formula shows that the value of $g$ that emerges in the $4 \mathrm{D}$ effective theory is not the one required by the gauge invariance. However, the disagreement is very small because 
of (3.37). Indeed, this is a general result: if $\mathcal{O}$ is an observable quantity in our 4D effective theory and $\mathcal{O}_{G I}$ the corresponding quantity in a gauge invariant theory, one can prov 19

$$
\frac{\mathcal{O}}{\mathcal{O}_{G I}}=1+O\left(\epsilon^{2}\right)
$$

Eqs. (4.63) and (4.64) represent the aforementioned result: the present model does not admit a $4 \mathrm{D}$ effective theory that can be written as a gauge theory with at most a spontaneous breaking of the gauge invariance. The reason why this happens is the presence of the weight functions in the 5D lagrangian, which are the only difference with respect to standard KK models. The same property is shared by higher dimensional gauge theories without fundamental scalars, but with some weight functions, which diverge when $|y| \rightarrow \infty$ [4].

As we commented before, the results of this subsection have been derived by neglecting higher order operators in (4.54). In Appendix B we show that the higher order operators do not modify these results in the semiclassical approximation.

\section{2 $5 \mathrm{D}$ versus $4 \mathrm{D}$ Higgs mechanism}

In this subsection we comment about the nature of the Higgs mechanism in our 5D model. As we pointed out, the main motivation of the present work is to present a model that relates the SSB of the gauge symmetry to a chiral asymmetry. However, one can wonder if the same mechanism may be realized by using a purely $4 \mathrm{D}$ language. In other words, is it possible to reproduce such a result first by constructing the $4 \mathrm{D}$ effective theory around an unstable vacuum and then by considering the SSB in such a theory 20 ?

The answer to this question is generally negative and, in order to understand why, we again consider the simple bosonic completion that we discussed in the present paper. The 4D effective theory approach to SSB requires to start with the unstable solution $\langle\phi\rangle=0$ in the presence of a small 21 but non vanishing $v$ in the Mexican hat potential. We observe that this set up can be equivalently achieved by setting $v=0$ in (3.24) and (3.25) and introducing a 5D gauge invariant and tachyonic mass term $+\mu^{2}|\phi|^{2}$ in the lagrangian, where $\mu^{2} \equiv 2 v^{2} \lambda$. Therefore, in this case, the light fermion modes are vector-like because the internal profile $f_{0}$ of the light vector mode is constant. Moreover, the $4 \mathrm{D}$ effective theory turns out to have an exact gauge invariance. If one now considers the Higgs mechanism in such a $4 \mathrm{D}$ theory the fermion spectrum will certainly remain chiral and, by definition, the gauge invariance will be spontaneously - not explicitly - broken.

This argument clearly shows that the $4 \mathrm{D}$ effective theory approach to SSB does not reproduce the exact values of all the observable quantities both in the fermionic and in the bosonic sector. However, we observe that in the bosonic sector this disagreement is very small because of (4.64); therefore it is not very surprising as we expect the $4 \mathrm{D}$ effective theory approach to SSB to be approximately correct only at energies much smaller than the mass of the first KK

\footnotetext{
${ }^{19}$ We checked the validity of Eq. (4.64) for all the interactions by using a method analogous to the one applied to show (4.63).

${ }^{20}$ In the following we shall call this approach the $4 D$ effective theory approach to $S S B$.

${ }^{21}$ Here $v$ small means $2 v^{2} \lambda \ll \mathcal{M}^{2}$.
} 
particles 22 . On the other hand, in the fermionic sector, this disagreement can be important as the distance $l$ between the left-handed and right-handed branes can be very large, leading to a non negligible chiral asymmetry.

\section{Non-Abelian Extensions}

In this section we comment that a relation between the SSB and the (low energy) chiral asymmetry can also be found in the presence of a non-Abelian gauge group $G$, by generalising in a natural way our previous analysis. We also discuss the case $G=S U(2) \times U(1)$ and some difficulties in constructing a realistic model.

\subsection{General non-Abelian gauge groups}

Here we start from a general compact Lie group $G$ with an arbitrary number of hermitian generators $T^{I}, I=1, \ldots, N$. The gauge field $A_{M}$ is a Lie Algebra valued vector field $\left(A_{M}=\right.$ $\left.A_{M}^{I} T^{I}, A_{M}^{\dagger}=A_{M}\right)$. We introduce a scalar $\phi$ in a non-trivial representation of $G$ and a scalar potential $V(\phi)$ that triggers the SSB $(\langle\phi\rangle \neq 0)$. We assume a bosonic action with the same form as $S_{B}$ in (3.20), but with $F_{M N}$ being a non-Abelian gauge field strength 23 and $\phi$ in general a multiplet. The covariant derivative of $\phi$ has now the form

$$
D_{M} \phi=\left(\partial_{M}+i g_{I} A_{M}^{I} T^{I}\right) \phi,
$$

where $g_{I}$ are the gauge constants of $G$ (in general we have more than one gauge constant). For the sake of simplicity, we take $\langle\phi\rangle$ to be constant, which is a legitimate set up.

In this more general case, the SSB contribution to the $5 \mathrm{D}$ vector boson mass terms in the $5 \mathrm{D}$ lagrangian is

$$
-\Delta_{S}<\phi>^{\dagger} g_{I} T^{I} g_{J} T^{J}<\phi>A_{M}^{I} A^{J M} \equiv-\Delta_{S} T^{I J} A_{M}^{I} A^{J M},
$$

where the constant matrix $T^{I J}$ can be diagonalised by a constant unitary transformation in the Lie algebra space:

$$
\mathcal{A}_{M}^{\beta}=U_{\beta I} A_{M}^{I},
$$

where $U$ is a unitary matrix. This definition also induces a redefinition of the generators: $\mathcal{T}^{\beta}=g_{I} T^{I} U_{I \beta}^{(-1)}$, where $U^{(-1)}$ is the inverse of $U$. We observe that the gauge fields $\mathcal{A}_{M}^{\beta}$ are not necessarily real and the generators $\mathcal{T}^{\beta}$ are not necessarily hermitian.

Also it is possible to add, like in the Abelian case, a gauge fixing term $\mathcal{L}_{G F}$ in the 5D lagrangian that removes the mixing between $4 \mathrm{D}$ vectors and $4 \mathrm{D}$ scalars:

$$
\mathcal{L}_{G F}=-\frac{\Delta}{2} \sum_{\beta}\left|\frac{1}{\Delta} \partial_{M}\left(\Delta \mathcal{A}^{\beta M}\right)-2 i \frac{\Delta_{S}}{\Delta}<\phi>^{\dagger}\left(\mathcal{T}^{\beta}\right)^{\dagger} \Omega\right|^{2},
$$

\footnotetext{
${ }^{22}$ Indeed, in this limit, such approach has proved to be correct by considering higher dimensional scalar theories [7].

${ }^{23}$ Here we assume the vector weight function $\Delta$ to be universal, namely to be the same function for all the simple factors in $G$. However, we could also consider different weight functions for different simple factors.
} 
where $\Omega \equiv \phi-\langle\phi\rangle$. Gauge fixing (5.67) represents the $R_{\xi}$ gauge (for $\xi=1$ ) in 5D warped models for general gauge groups and, by generalizing in a straightforward way Appendix $\mathrm{A}$, it is easy to show that it is indeed a legitimate choice.

With this gauge fixing the EOM of the spin-1 fields are

$$
\frac{1}{\Delta} \partial_{M}\left(\Delta \partial^{M} \mathcal{A}^{a \mu}\right)=0, \quad \frac{1}{\Delta} \partial_{M}\left(\Delta \partial^{M} \mathcal{A}^{\hat{a} \mu}\right)=2 t_{\hat{a}} \frac{\Delta_{S}}{\Delta} \mathcal{A}^{\hat{a} \mu},
$$

where $\mathcal{A}_{\mu}^{a}$ correspond to the unbroken symmetries $\left(\mathcal{T}^{a}<\phi>=0\right), \mathcal{A}_{\mu}^{\hat{a}}$ correspond to the broken symmetries $\left(\mathcal{T}^{\hat{a}}<\phi>\neq 0\right)$,

$$
t_{\hat{a}} \equiv\left|\mathcal{T}^{\hat{a}}<\phi>\right|^{2}
$$

and in (5.68) the index $\hat{a}$ is not contracted. It is now clear that the profiles of the lightest 4D vector fields coming from $\mathcal{A}_{\mu}^{a}$ are generically constant along the extra dimension, whereas the profiles of the lightest $4 \mathrm{D}$ vector fields coming from $\mathcal{A}_{\mu}^{\hat{a}}$, have generically a non-trivial shape in the broken phase and can be localized on a particular point of the extra dimensions, say $y=0$, by choosing suitable weight functions. Of course, like in the Abelian case, the non-trivial profiles will reduce to constant ones in the unbroken limit $\langle\phi\rangle \rightarrow 0$.

The 5D fermion field $\psi$ will also belong to a non-trivial representation of $G$ and, if we assume a fermion action of the form (24.1), we can again localize the lightest modes of $\psi_{L}$ and $\psi_{R}$ on different points of the extra dimension, say $y=0$ and $y=l$, by choosing a suitable background domain wall $\varphi$, e.g. Eq. (2.12). Analogously to the Abelian case, the 4D couplings between the light vectors and fermions can be obtained by integrating over the extra dimensions the following operators

$$
\left[\overline{\psi_{L}} \gamma^{\mu} \mathcal{A}_{\mu}^{a} \mathcal{T}_{f}^{a} \psi_{L}+\overline{\psi_{R}} \gamma^{\mu} \mathcal{A}_{\mu}^{a} \mathcal{T}_{f}^{a} \psi_{R}+\overline{\psi_{L}} \gamma^{\mu} \mathcal{A}_{\mu}^{\hat{a}} \mathcal{T}_{f}^{\hat{a}} \psi_{L}+\overline{\psi_{R}} \gamma^{\mu} \mathcal{A}_{\mu}^{\hat{a}} \mathcal{T}_{f}^{\hat{a}} \psi_{R}\right]_{l i g h t}
$$

where the label "light" means that we are selecting only the light modes in the various KK expansions. Therefore, the fermion representation of the residual gauge group, with generators $\mathcal{T}^{a}$ will certainly be vector-like, whereas the interactions between fermions and light (but massive) vector bosons will be chiral. Such chiral asymmetry will reduce to zero in the unbroken limit for the same reason as it does in the simple Abelian case.

Here we also observe that we cannot expect the low energy $4 \mathrm{D}$ effective theory to have at most a spontaneous breaking of the gauge symmetry. This is because the simple 5D warped Higgs model that we analyzed before (and all the non-Abelian generalizations that reduce to it via a consistent truncation) represent explicit counterexamples.

\subsection{The $S U(2) \times U(1)$ case}

As a particular case, here we consider in more detail the electroweak cas $25 G=S U(2) \times U(1)$. Let us denote with $W_{M}=W_{M}^{I} \frac{\tau^{I}}{2}$, where $\tau^{I}$ are the Pauli matrices, and $B_{M}$ the $S U(2)$ and

\footnotetext{
${ }^{24}$ Now we have $D_{M} \psi=\left(\partial_{M}+i \mathcal{A}_{M}^{\beta} \mathcal{T}_{f}^{\beta}\right) \psi$, where $T_{f}^{\beta}$ are the generators in the fermion representation.

${ }^{25}$ The extension to the $\mathrm{SM}$ gauge group $S U(3) \times S U(2) \times U(1)$ is trivial as the $S U(3)$ factor is not broken.
} 
the $U(1)$ gauge fields respectively. We also choose the $5 \mathrm{D}$ scalar field to transform as $\phi \sim \mathbf{2}_{1 / 2}$ under $S U(2) \times U(1)$. Therefore, we have

$$
D_{M} \phi=\left(\partial_{M}+i g W_{M}+i \frac{g^{\prime}}{2} B_{M}\right) \phi
$$

where $g$ and $g^{\prime}$ are the gauge constants of $S U(2)$ and $U(1)$ respectively. In this case the bosonic action is

$$
S_{B}=\int d^{5} X\left\{-\frac{\Delta}{4}\left(W_{M N} W^{M N}+B_{M N} B^{M N}\right)-\Delta_{S}\left[\left(D_{M} \phi\right)^{\dagger} D^{M} \phi+V(\phi)\right]\right\},
$$

where $W_{M N}$ and $B_{M N}$ are the field strengths of $W_{M}$ and $B_{M}$ respectively and $V(\phi)$ is a scalar potential that triggers the following VEV

$$
<\phi>=\left(\begin{array}{l}
0 \\
v
\end{array}\right),
$$

where $v$ is a real number.

Like in the SM, we now introduce

$$
\begin{aligned}
W_{M}^{ \pm} & =\frac{1}{\sqrt{2}}\left(W_{M}^{1} \pm i W_{M}^{2}\right), \\
Z_{M} & =\cos \theta W_{M}^{3}-\sin \theta B_{M}, \\
\gamma_{M} & =\sin \theta W_{M}^{3}+\cos \theta B_{M},
\end{aligned}
$$

where $\theta$ is defined by $g \sin \theta=g^{\prime} \cos \theta$. We identify the lightest modes from $W_{\mu}^{ \pm}, Z_{\mu}$ and $\gamma_{\mu}$ with the $W^{ \pm}, Z$ and photon vector bosons respectively. The corresponding profiles can be computed through Eqs. (5.68) and (5.69); in our case we have

$$
t_{+}=t_{-}=\frac{1}{4} v^{2} g^{2}, \quad t_{Z}=\frac{1}{4} v^{2}\left(g^{2}+g^{\prime 2}\right),
$$

where $t_{ \pm}$and $t_{Z}$ are the parameters defined in (5.69) for $W_{\mu}^{ \pm}$and $Z_{\mu}$ respectively. The relation $g \sin \theta=g^{\prime} \cos \theta$ guarantees that the linearized EOM for $\gamma_{\mu}$ is simply

$$
\frac{1}{\Delta} \partial_{M}\left(\Delta \partial^{M} \gamma^{\mu}\right)=0
$$

Therefore, in the broken phase, we can localize the lightest modes of $W_{\mu}^{ \pm}$and $Z_{\mu}$ on some point of the extra dimension, say $y=0$, with a suitable choice of the weight functions. In the unbroken limit $(v \rightarrow 0)$ these profiles will go to constants. On the other hand, the lightest mode of $\gamma_{\mu}$ is generically delocalized and massless both for $v \neq 0$ and $v=0$, because the corresponding gauge symmetry is unbroken.

Now we consider a 5D fermion field 26 transforming as $\psi \sim \mathbf{2}_{-1 / 2}$. We can decompose $\psi$ as

$$
\psi=\left(\begin{array}{l}
\nu \\
e
\end{array}\right),
$$

\footnotetext{
${ }^{26}$ Here we understand a flavor index and, therefore, the number of family is generic.
} 
and identify the lightest mode of $e$ and $\nu$ with an electron and a neutrino respectively. Since we have a $5 \mathrm{D}$ space-time we necessarily have both the $4 \mathrm{D}$ chiralities: $e=e_{L}+e_{R}$ and $\nu=\nu_{L}+\nu_{R}$. If we again localize the left-handed fermions on $y=0$ and the right-handed ones on $y=l$, we should be able to reproduce realistic couplings between fermions and $W^{ \pm}$: the interaction between $W^{ \pm}$and the left-handed fermions can be achieved by choosing a suitable value of $g$, whereas the coupling between $W^{ \pm}$and the right-handed fermions is suppressed by construction. The coupling between $\gamma$ and the fermions may also be realistic: we are free to adjust the coupling between $e$ and $\gamma_{\mu}$ by properly choosing $g^{\prime}$ and the interaction between $\nu$ and $\gamma_{\mu}$ is automatically zerd27. The only interactions between fermions and vector bosons which certainly cannot be reproduced in this simple framework are those involving $Z$. Indeed, if we do not modify our set up, the coupling between $Z$ and the right-handed electron will turn out to be suppressed as the respective profiles are localized on two different points of the extra dimension, which we take far away each other. We also observe that this mismatch is due to the fact that in the SM we have $g_{S M}^{\prime} \neq 0$ and so $Z$ interacts non trivially with the right-handed electron. Therefore, our simple model reproduce the correct fermion-vector interactions in the limit $g^{\prime} \rightarrow 0$.

To conclude, the minimal implementation of our mechanism can reproduce phenomenologically correct interactions between the fermions and $\left\{W^{ \pm}, \gamma\right\}$, but is not general enough to be realistic. This is true not only because the correct interactions of $Z$ cannot be reproduced, but also because it seems difficult, at least in this simple set up, to achieve the correct fermion and vector boson masses. Indeed, we could think to introduce some Yukawa interactions in the 5D lagrangian in order to obtain a realistic fermion mass spectrum, but then we would probably introduce a non-universality in the gauge interactions of different families. Moreover, the spectrum of the vector bosons is also problematic because the value of the $t$-parameters appearing in (5.75) are not necessarily proportional to the masses of the vector bosons (like in the SM). Indeed, these masses must be computed by solving the Eqs (5.68) and therefore will depend in general on the shape of the wave functions.

However, we consider the present discussion interesting as we found a relation between the chiral asymmetry and the Higgs mechanism in a model that resembles the electroweak theory in the low energy limit. We plan to study more general and possibly realistic implementation of our mechanism in a future phenomenological extension of the present theoretical work.

\section{Conclusions and Outlook}

In this paper we have proposed a mechanism that relates the chiral asymmetry to the 5D Higgs mechanism, which generates masses for the low energy degrees of freedom. To illustrate the basic idea we have analyzed a 5D fermion and, as a bosonic completion, we have considered a simple 5D warped Higgs model. Such mechanism exploits the fact that the 4D gauge field profile along the extra dimension is generally constant if the gauge symmetry is unbroken, but it can be peaked around some point, for example $y=0$, in the broken case. This point also represents the $4 \mathrm{D}$ world where fermions with a given chirality live, whereas the other chirality can be localized on another point $y=l$, by means of a domain wall configuration.

\footnotetext{
${ }^{27}$ This is a consequence of our choice $\psi \sim \mathbf{2}_{-1 / 2}$, like in the SM.
} 
A specific feature of our mechanism is that, in the case $v \neq 0$, the bosonic action of the $4 \mathrm{D}$ effective theory generically cannot be written as a gauge invariant action with at most a spontaneous breaking of the gauge symmetry. This statement has been proved by choosing some specific values of the weight functions, which may have their origin in warped compactification of theories of gravity or supergravity. Indeed, for these specific values, all the bosonic profiles along the extra dimension are simply 1D harmonic oscillator wave functions and, therefore, many observable quantities can be explicitly computed. Then, we have considered two possible physical definitions of the 4D gauge constant, which must coincide in a gauge invariant theory. The first definition can be obtained from the decay amplitude of the physical Higgs field $\omega_{1}$ into two light vector bosons $V$, whereas the second one comes from the scattering $\omega_{1}, V \rightarrow \omega_{1}, V$. These coupling constants turned out to be different, even if their relative difference is a very small quantity, of the order of the square of the ratio between the electroweak scale and the lightest heavy mode mass. Remarkably, the $4 \mathrm{D}$ effective theory becomes gauge invariant, at least at the semiclassical level, as $v \rightarrow 0$ that is exactly the limit in which the chiral asymmetry disappears.

As a consequence of the aforementioned results, the Higgs mechanism, which we triggered in order to achieve our purpose, cannot be described by a purely $4 \mathrm{D}$ method, that we called $4 \mathrm{D}$ effective theory approach to SSB and defined in Subsection 4.2. Indeed, the latter method is expected to be correct at the leading order in $\epsilon$ in standard KK theories, but in our model breaks down because of the presence of an additional parameter, $l$. By taking into account $\epsilon^{2}$ corrections, such a method turned out to be incorrect even without fermions. This is not very surprising, because the $4 \mathrm{D}$ effective theory is expected to be correct only at energies much smaller than the heavy KK mode mass, but is still interesting because in our model the $\epsilon^{2}$ corrections are the first non-trivial corrections.

Moreover, we commented that a relation between the chiral asymmetry and the Higgs mechanism can also emerge in non-Abelian generalizations of the warped Higgs model with fermions, which we have previously analyzed. We showed that the unbroken directions in the Lie algebra space correspond to vector-like interactions in the low energy theory, whereas the remaining directions present a chiral asymmetry. As an explicit non-Abelian example we considered the electroweak group $S U(2) \times U(1)$ and we obtained, by using a simple set up, a low energy limit that resembles - but does not entirely reproduce - the standard electroweak theory. Some of the reasons why the simple implementation that we considered is not realistic are the fact that the correct interactions of $\mathrm{Z}$ are not reproduced and it seems difficult to obtain realistic fermion and vector boson masses.

An interesting development of the present work can be the extension of the simple implementation presented in this paper to a theory which exactly reduces to the SM at low energies. For example, extensions of this type might be the introduction of more than two branes, general Yukawa couplings and general weight functions in the higher dimensional model. Moreover, it would be nice to have an embedding of this type of models in a more fundamental theory which includes gravity. Indeed, this may lead to a dynamical origin of the domain wall configuration $\varphi$ and the weight functions. Finally, another interesting direction is the complete calculation of the heavy mode contribution to the $4 \mathrm{D}$ effective theory, including the effect of the heavy fermion fields: an exact cancellation of the chiral anomaly may happen as in previous works [18, 10] and some observational effects could emerge [19]. 
Acknowledgments. We would especially like to thank A. Boyarsky, Y. Burnier, R. Rattazzi, O. Ruchayskiy, A. Wulzer and K. Zuleta for interesting and useful communications. This work was supported by the Tomalla Foundation.

\section{Appendix}

\section{A $\quad R_{\xi}$ Gauges in 5D Warped Models}

In this appendix we show that the following clas 28 of gauge fixing terms

$$
\mathcal{L}_{G F}=-\frac{\Delta}{2 \xi}\left[\frac{1}{\Delta} \partial_{M}\left(\Delta A^{M}\right)+\sqrt{2} e v \xi \frac{\Delta_{S}}{\Delta} \eta\right]^{2},
$$

represents possible gauge fixings. In analogy with the $4 \mathrm{D}$ case, we refer to them as $R_{\xi}$ gauges. More precisely, here we prove that, for a given initial configuration $\mathcal{G}_{i}$ of the gauge function $\mathcal{G} \equiv(1 / \Delta) \partial_{M}\left(\Delta A^{M}\right)+\sqrt{2} e v \xi\left(\Delta_{S} / \Delta\right) \eta$ and for a given arbitrary space time function $\varepsilon(X)$, we can always find a gauge transformation of the form (3.21) such that $\mathcal{G}_{i} \rightarrow \mathcal{G}_{i}+\varepsilon$. In the rest we assume $\varepsilon$ to be infinitesimal as the general case can be addressed by considering an infinite number of infinitesimal transformations.

Therefore, we have to explicitly find a solution to the following equation

$$
-\frac{1}{\Delta} \partial_{M}\left(\Delta \partial^{M} \alpha\right)+e^{2} K \alpha=\varepsilon,
$$

where $K \equiv 2 v \xi \frac{\Delta_{S}}{\Delta}(v+\sigma / \sqrt{2})$. In (A.79) we used $\operatorname{Im}\left(e^{i e \alpha} \phi\right)=e \alpha(v+\sigma / \sqrt{2})+\eta / \sqrt{2}$, which is valid for an infinitesimal value of $\alpha$. We can find a solution to Eq. (A.79) by using the perturbation theory with respect to $e^{2}$; a non-perturbative treatment of Eq. (A.79) is beyond our purposes since we always used a perturbative approach in this paper 29 .

We start by considering the unperturbed $(e=0)$ equation:

$$
-\frac{1}{\Delta} \partial_{M}\left(\Delta \partial^{M} \alpha_{0}\right)=\varepsilon
$$

A solution $\alpha_{0}$ to this equation can be written in terms of the Green's function $G_{0}\left(X, X^{\prime}\right)$ for the operator $-\frac{1}{\Delta} \partial_{M}\left(\Delta \partial^{M}\right.$ :

$$
G_{0}\left(X, X^{\prime}\right)=\sum_{n} \int \frac{d^{4} q}{(2 \pi)^{4}} \frac{1}{q^{2}+\lambda_{n}} e^{i q\left(x-x^{\prime}\right)} \psi_{n}(y) \psi_{n}^{*}\left(y^{\prime}\right),
$$

where $\left\{\psi_{n}\right\}$ is a complete set of eigenfunctions of the operator $-\Delta^{-1} \partial_{y}\left(\Delta \partial_{y}\right.$ and $\lambda_{n}$ the corresponding eigenvalues. We have already studied this basis in Subsection 3.2 .

$$
\psi_{n}=\lim _{v \rightarrow 0} f_{n} \quad \text { and } \quad \lambda_{n}=\lim _{v \rightarrow 0} M_{n}^{2} .
$$

\footnotetext{
${ }^{28}$ Since we can do so without much expense, here we keep a generic value of the constant $\xi$.

${ }^{29}$ We thank Riccardo Rattazzi for a discussion on this issue.
} 
A solution to Eq. (A.80) can be now written as follows

$$
\alpha_{0}=\int d^{5} X_{1} G_{0}\left(X, X_{1}\right) \varepsilon\left(X_{1}\right)
$$

whereas a complete solution to Eq. (A.79) can be expressed in terms of a Taylor series with respect to $e^{2}$, that is $\alpha=\sum_{j=0}^{+\infty} \alpha_{j}$, where $\alpha_{0}$ is given in (A.82) and

$$
\begin{aligned}
\alpha_{1}(X)= & -e^{2} \int d^{5} X_{1} G_{0}\left(X, X_{1}\right) K\left(X_{1}\right) \int d^{5} X_{2} G_{0}\left(X_{1}, X_{2}\right) \varepsilon\left(X_{2}\right), \\
\ldots, \alpha_{j}(X)= & (-1)^{j} e^{2 j} \int d^{5} X_{1} G_{0}\left(X, X_{1}\right) K\left(X_{1}\right) \int d^{5} X_{2} G_{0}\left(X_{1}, X_{2}\right) K\left(X_{2}\right) \times \ldots \times \\
& \int d^{5} X_{j} G_{0}\left(X_{j-1}, X_{j}\right) K\left(X_{j}\right) \int d^{5} X_{j+1} G_{0}\left(X_{j}, X_{j+1}\right) \varepsilon\left(X_{j+1}\right) .
\end{aligned}
$$

\section{B Higher Dimensional Operators}

Here we analyze the higher dimensional gauge invariant operators in (4.54) that could modify (after SSB) the operators $V_{\mu} V^{\mu}, V_{\mu} V^{\mu} \omega_{1}$ and $V_{\mu} V^{\mu} \omega_{1}^{2}$. Indeed, other operators cannot ruin the argument given in Section 4 that leads to a small gauge symmetry breaking in the effective theory.

Such higher dimensional operators should contain a term with no derivatives and where $V_{\mu}$ appears in the form $V_{\mu} V^{\mu}$. We also observe that the only way to construct gauge invariant operators which contain $V_{\mu}$ is through $D_{\mu} \omega$ and $V_{\mu \nu}$, but it is impossible to construct an operator without derivatives if we use $V_{\mu \nu}$. Therefore, we should start from the following type of gauge invariant operators:

$$
\left(\omega^{\dagger} \omega\right)^{p}\left(D_{\mu} \omega\right)^{\dagger} D^{\mu} \omega
$$

where $p=1,2,3, \ldots$. Indeed, if we started from an operator which contains more than two covariant derivatives $D_{\mu} \omega$ and then extracted a term where $V_{\mu}$ appears in the form $V_{\mu} V^{\mu}$, we would necessarily have some derivatives as well.

Let us show that $(\mathrm{B} .83)$ cannot be derived from our $5 \mathrm{D}$ theory, whose action is given in (3.25). To construct (B.83), among other things, we need the following operator:

$$
\omega_{1}^{2 p} \partial_{\mu} \omega_{1} \partial^{\mu} \omega_{1}
$$

which follows from (B.83) when we select $\omega_{1}$ in the expansion $\omega=\hat{v}+\left(\omega_{1}+i \omega_{2}\right) / \sqrt{2}$, and $\partial_{\mu} \omega_{1}$ in the expansion $D_{\mu} \omega=\left(\partial_{\mu}+i \hat{e} V_{\mu}\right)\left[\hat{v}+\left(\omega_{1}+i \omega_{2}\right) / \sqrt{2}\right]$. Remember also $\omega_{1} \equiv \sigma_{1}$.

We prove now that operators of the form (B.84) can appear in our model only through loop corrections, which we do not include in the present analysis. First notice that, in order to construct a tree-level contribution to (B.84), we need at least one vertex containing only one heavy line (this is because in tree-diagrams we must have some vertices where an internal line ends). Vertices with only one heavy line and light lines of the $\sigma_{1}$-type only come from the following interactions in 30 (3.25):

$$
\int d^{5} X\left(-\sqrt{2} v \lambda \Delta_{S} \sigma^{3}-\frac{\lambda}{4} \Delta_{S} \sigma^{4}\right)
$$

\footnotetext{
${ }^{30}$ Though (B.85) does not contain derivatives as in (B.84), they arise after expanding over the momentum.
} 
The first and second terms in (B.85) lead to two classes of $4 \mathrm{D}$ interactions of the required type, whose coupling constants are respectively proportional to

$$
\int d y \Delta_{S}^{-1 / 2} \chi_{\sigma 1}^{2} \chi_{\sigma n}, \quad \text { and } \quad \int d y \Delta_{S}^{-1} \chi_{\sigma 1}^{3} \chi_{\sigma n}
$$

where $n>1$. Now, by using $\chi_{\sigma 1} \propto \Delta_{S}^{1 / 2}$ and the orthogonality between $\chi_{\sigma 1}$ and $\chi_{\sigma n}$, with $n>1$, we have

$$
\int d y \Delta_{S}^{-1 / 2} \chi_{\sigma 1}^{2} \chi_{\sigma n} \propto \int d y \chi_{\sigma 1} \chi_{\sigma n}=0
$$

and

$$
\int d y \Delta_{S}^{-1} \chi_{\sigma 1}^{3} \chi_{\sigma n} \propto \int d y \chi_{\sigma 1} \chi_{\sigma n}=0 .
$$

Therefore, we cannot construct (at the semiclassical level) a gauge invariant operator of the form (B.83) in our model. We conclude that the argument provided in Section 4 is valid even if we take into account higher order operators in (4.54).

\section{References}

[1] N. S. Manton, "Fermions And Parity Violation In Dimensional Reduction Schemes," Nucl. Phys. B 193 (1981) 502.

[2] V. A. Rubakov and M. E. Shaposhnikov, "Do We Live Inside A Domain Wall?," Phys. Lett. B 125 (1983) 136.

[3] G. R. Dvali, S. Randjbar-Daemi and R. Tabbash, "The origin of spontaneous symmetry breaking in theories with large extra dimensions," Phys. Rev. D 65 (2002) 064021 arXiv:hep-ph/0102307.

[4] M. E. Shaposhnikov and P. Tinyakov, "Extra dimensions as an alternative to Higgs mechanism?," Phys. Lett. B 515 (2001) 442 arXiv:hep-th/0102161.

[5] V. A. Rubakov and M. E. Shaposhnikov, "Extra Space-Time Dimensions: Towards A Solution To The Cosmological Constant Problem," Phys. Lett. B 125 (1983) 139.

S. Randjbar-Daemi and C. Wetterich, "Kaluza-Klein Solutions With Noncompact Internal Spaces," Phys. Lett. B 166 (1986) 65.

[6] L. Randall and R. Sundrum, "An alternative to compactification," Phys. Rev. Lett. 83 (1999) 4690 arXiv:hep-th/9906064.

[7] S. Randjbar-Daemi, A. Salvio and M. Shaposhnikov, "On the decoupling of heavy modes in Kaluza-Klein theories," Nucl. Phys. B 741 (2006) 236 arXiv:hep-th/0601066.

[8] A. Salam and J. A. Strathdee, "On Kaluza-Klein Theory," Annals Phys. 141 (1982) 316. S. Randjbar-Daemi, A. Salam and J. A. Strathdee, "Spontaneous Compactification In Six-Dimensional Einstein-Maxwell Theory," Nucl. Phys. B 214 (1983) 491. 
[9] S. Randjbar-Daemi and M. Shaposhnikov, "A formalism to analyze the spectrum of brane world scenarios," Nucl. Phys. B 645 (2002) 188 [arXiv:hep-th/0206016].

[10] D. B. Kaplan and M. Schmaltz, "Domain Wall Fermions and the Eta Invariant," Phys. Lett. B 368 (1996) 44 arXiv:hep-th/9510197].

[11] C. D. Fosco and R. C. Trinchero, "Domain walls and dimensional reduction," Nucl. Phys. B 563 (1999) 533 arXiv:hep-th/9908034.

[12] S. J. Huber and Q. Shafi, "Higgs mechanism and bulk gauge boson masses in the RandallSundrum model," Phys. Rev. D 63 (2001) 045010 [arXiv:hep-ph/0005286].

K. Ghoroku and A. Nakamura, "Massive vector trapping as a gauge boson on a brane," Phys. Rev. D 65 (2002) 084017 arXiv:hep-th/0106145].

I. I. Kogan, S. Mouslopoulos, A. Papazoglou and G. G. Ross, "Multi-localization in multibrane worlds," Nucl. Phys. B 615 (2001) 191 arXiv:hep-ph/0107307.

G. Burdman, "Constraints on the bulk standard model in the Randall-Sundrum scenario," Phys. Rev. D 66 (2002) 076003 arXiv:hep-ph/0205329].

B. Batell and T. Gherghetta, "Localized U(1) gauge fields, millicharged particles, and holography," Phys. Rev. D 73 (2006) 045016 arXiv:hep-ph/0512356].

[13] S. L. Parameswaran, S. Randjbar-Daemi and A. Salvio, "Gauge fields, fermions and mass gaps in 6D brane worlds," Nucl. Phys. B 767, 54 (2007) arXiv:hep-th/0608074.

S. L. Parameswaran, S. Randjbar-Daemi and A. Salvio, "Stability and Negative Tensions in 6D Brane Worlds," arXiv:0706.1893 [hep-th].

[14] A. Muck, A. Pilaftsis and R. Ruckl, "Minimal higher-dimensional extensions of the standard model and electroweak observables," Phys. Rev. D 65 (2002) 085037 arXiv:hep-ph/0110391.

[15] H. Nicolai and C. Wetterich, "On The Spectrum Of Kaluza-Klein Theories With Noncompact Internal Spaces," Phys. Lett. B 150 (1985) 347.

G. W. Gibbons and D. L. Wiltshire, "Space-Time As A Membrane In Higher Dimensions," Nucl. Phys. B 287, 717 (1987) arXiv:hep-th/0109093].

A. Kehagias, "On non-compact compactifications with brane worlds," arXiv:hep-th/9911134.

[16] J. M. Cornwall, D. N. Levin and G. Tiktopoulos, "Derivation Of Gauge Invariance From High-Energy Unitarity Bounds On The S Matrix," Phys. Rev. D 10 (1974) 1145 [Erratumibid. D 11 (1975) 972].

[17] A. Salvio, "4D effective theory and geometrical approach," AIP Conf. Proc. 881 (2007) 58 arXiv:hep-th/0609050.

A. Salvio, "Aspects of physics with two extra dimensions," author's Ph.D. thesis arXiv:hep-th/0701020.

[18] C. G. Callan and J. A. Harvey, "Anomalies And Fermion Zero Modes On Strings And Domain Walls," Nucl. Phys. B 250 (1985) 427. 
[19] S. Y. Khlebnikov and M. E. Shaposhnikov, "Extra space-time dimensions: towards a solution to the strong CP problem," Phys. Lett. B 203 (1988) 121.

S. Khlebnikov and M. Shaposhnikov, "Brane-worlds and theta-vacua," Phys. Rev. D 71 (2005) 104024 arXiv:hep-th/0412306.

A. Boyarsky, O. Ruchayskiy and M. Shaposhnikov, "Anomalies as a signature of extra dimensions," Phys. Lett. B 626 (2005) 184 arXiv:hep-ph/0507195].

A. Boyarsky, O. Ruchayskiy and M. Shaposhnikov, "Observational manifestations of anomaly inflow," Phys. Rev. D 72 (2005) 085011 arXiv:hep-th/0507098.

S. Khlebnikov, "Massive Schwinger model with a finite inductance: theta-(in)dependence, the U(1) problem, and low-energy theorems," Phys. Rev. D 74 (2006) 085007 arXiv:hep-th/0608070. 